\title{
Setting the stage for CFTR modulator studies in infants
}

\author{
Peter J F M Merkus
}

Because irreversible damage to the cystic fibrosis (CF) lung is already observed in infancy, a need has arisen to assess and monitor lung function early in life. Infant lung function testing (ILFT) is non-invasive, does not require radiation or anaesthesia and can be repeated frequently; therefore, development, standardisation and validation of techniques, protocols and equipment have received priority. Thanks to the hard work of many researchers across the globe during the last few decades, we are now able to study and compare development of lung function in the youngest during health and disease. ${ }^{1}$ Through

Correspondence to Peter J F M Merkus, Department of Pediatrics, Division of Respiratory Medicine and Allergology, Route 804 Post: 804, Amalia Children's Hospital, Radboud University Medical Centre, Po Box 9101, Nijmegen 6500 HB, The Netherlands; peter.merkus@radboudumc.nl standardisation, and the use of appropriate reference populations, we can now collate data from different centres to find reliable answers to basic questions on developmental physiology ${ }^{12}$ and to study lung function growth in chronic lung disease. ${ }^{3-5}$

The study by Nguyen et $a l^{6}$ illustrates the value of such collaboration. Rather than competing with one another, the $6 \mathrm{CF}$ centres of The London CF Collaboration (LCFC) shared protocols for treatment and follow-up. As a result, a relatively large group of infants with CF treated, diagnosed through NewBorn Screening (NBS) according to a standardised regimen could be analysed in relatively short period of time. ILFT was assessed in one specialised centre with sufficient experience and capacity to carry out longitudinal ILFT measurements. Moreover, healthy controls of about the same age were measured as well, serving as an adequate control group.
Lung function was below average at 3 months of age but remained stable or improved in NBS CF infants during the first year of life, and deficits at 1 year were considerably smaller than observed one decade earlier in either screened or clinically diagnosed infants, possibly suggesting that current treatment is more effective in preventing early loss of lung function.

Though the authors state that the study provides 'evidence regarding the natural changes that can occur over time...', this study does not really reflect the natural course of the disease: these patients received treatment according to guidelines and local protocols including flucloxacillin prophylaxis and received intensified treatment when considered necessary by their physicians based on the clinical presentation, or-presumably-based on the ILFT results. Nevertheless, consortia like this collating ILFT data are indispensable when evaluating symptomatic treatment such as mucolytics, antibiotics or even smart nebulisers. 17

Especially when international trials can be conducted through consortia we will be able to make much more progress in this world of CF, in which there is so little 
time to waste. Similar studies conducted in Australia, however, yielded quite different results. Based on the AREST-CF data, ${ }^{8} 9$ it appeared that initial lung function in NBS CF infants was normal or nearly normal, followed by a subsequent decline in the first year of life. What could have caused such differences? Well, obviously many confounders may play a role that cannot be controlled for, such as altitude and climate, exposures to air pollution and parental smoking, adherence to treatment, type of antibiotic prophylaxis if any, first contact with Pseudomonas aeruginosa, in addition to technical differences such as pressures applied, hardware and software, and measurement protocols. Also, genotypes may explain these differences but genotype distribution seemed similar in the LCFC and the AREST CF cohorts: $61 \%$ of the LCFC cohort was homozygous dF508 compared with 50\% and $62 \%$ in the 2008 and 2011 Australian cohorts (Thanh Diem Nguyen, personal communication). So a good explanation for the differences in results from the two populations remains lacking.

Based on the LCFC data, the authors concluded that about 85 unselected newborn screened infants/arm are required for intervention trials to have sufficient power to detect the relatively small differences that can be expected in such trials. Moreover, if 'higher risk'infants with diminished ILFT at 3 months of age-were to be included selectively, larger differences may be detectable with smaller numbers. ${ }^{6}$ However, is that really the case? It is also possible that diminished ILFT at 3 months of age simply reflects biology, or early irreversible loss of airway function, leaving little room for improvement. And, furthermore: should we only focus on lung function as an end point? Clearly, lung function is a predictor of long-term prognosis, but there are also other pulmonary end points and even other target organs to consider in this systemic disease. And especially when we enter the era of cystic fibrosis transmembrane regulator (CFTR) modulators, other end points will have to be taken into account because they may be even more relevant or sensitive than lung function.

Lung imaging, especially using CT scans, is not only a suitable tool to assess lung growth $^{10}$ or parenchymal dimensions, ${ }^{11}$ but is also an increasingly popular end point to monitor CF lung disease. ${ }^{12-14}$ Indeed, the same London CF consortium also studied the added value of CT scans in monitoring early CF lung disease. ${ }^{15}$ And while Australian researchers concluded that chest CT scans provide relevant and sensitive additional information about early CF lung damage, the LCFC group found only mild structural changes and concluded that chest CT was of questionable value, also because of large interobserver and intraobserver agreements. ${ }^{15}$ However, that conclusion may be premature. Before it is concluded that CT scans have no role in monitoring early CF lung disease, efforts should be made to optimise the quality and sensitivity of scoring systems. Possibly, a modified and validated Brody II scoring system may help us ${ }^{16}$ where other scoring systems do not.

But at the same time, we should also think about additional end points that are reliable, acceptable, sensitive and feasible enough for future multicentre CFTR modulator trials. We know that lung function is poorly correlated to the sweat test $^{17}$ and improvement of the sweat test is only correlated with CFTR function below $40 \%$ of normal activity. ${ }^{18}$ Of the two tests that assess CFTR function in vivo, measuring nasal potential differences is technically difficult, requires good patient cooperation and may be insensitive to small changes in CFTR function. Therefore, measuring intestinal current measurements may be the best candidate for this purpose, ${ }^{19}$ but such measurements are somewhat invasive, not patientfriendly and time-consuming.

With the new classes of therapeutic compounds, it may be wise to also widen the scope and look at the function of the immune system. Innate immunity is affected by $\mathrm{CF}^{20}{ }^{21}$ and CFTR modulators may improve or normalise innate immunity. If this is the case, they may improve the number of exacerbations, affect the changes of the respiratory microbiome and long-term prognosis. So monitoring innate immunity might be an end point at least as important as monitoring structure and function of the lung.

Hence, with the novel CFTR modulator drugs underway, we should not only improve sensitivity and quality of end points but also seriously consider which additional end points should be developed and added to the clinical trials, next to the traditional (I)LFT. However, in the large majority of CF infants, administration of CFTR modulators will not be a part of the daily routine for the next 5 years or so and therefore, ILFT will remain a cornerstone outcome measure, preferably measured in well-organised consortia that are able to deliver highquality data based on large groups of infants with CF.

Competing interests None.
Provenance and peer review Commissioned; internally peer reviewed.

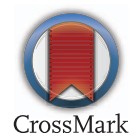

To cite Merkus PJFM. Thorax 2014;69:888-890.

Published Online First 6 August 2014

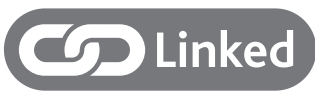

http://dx.doi.org/10.1136/thoraxjnl-2013-204023

Thorax 2014;69:888-890.

doi:10.1136/thoraxjnl-2013-204248

\section{REFERENCES}

1 Rosenfeld M, Allen J, Arets BH, et al. An official American Thoracic Society workshop report: optimal lung function tests for monitoring cystic fibrosis, bronchopulmonary dysplasia, and recurrent wheezing in children less than 6 years of age. Ann Am Thorac Soc 2013;10:S1-S11.

2 Chang DV, Tiller CJ, Kisling JA, et al. Membrane and capillary components of lung diffusion and pro-angiogenic cells in infants. Eur Respir $J$ 2014:43:497-504

3 Castillo A, Llapur CJ, Martinez T, et al. Measurement of single breath-hold carbon monoxide diffusing capacity in healthy infants and toddlers. Pediatr Pulmonol 2006;41:544-50.

4 Gappa M, Stocks J, Merkus P. Lung growth and development after preterm birth: further evidence. Am J Respir Crit Care Med 2003;168:399.

5 Sly PD, Brennan S, Gangell C, et al. Lung disease at diagnosis in infants with cystic fibrosis detected by newborn screening. Am J Respir Crit Care Med 2009;180:146-52.

6 Nguyen TT, Thia LP, Hoo AF, et al. on behalf of the London Cystic Fibrosis Collaboration (LCFC). Evolution of lung function during the first year of life in newborn screened cystic fibrosis infants. Thorax 2014;69:910-17.

7 Bakker EM, Volpi S, Salonini E, et al. Improved treatment response to dornase alfa in cystic fibrosis patients using controlled inhalation. Eur Respir J 2011;38:1328-35.

8 Linnane BM, Hall GL, Nolan G, et al. Lung function in infants with cystic fibrosis diagnosed by newborn screening. Am J Respir Crit Care Med 2008;178:1238-44.

9 Pillarisetti $\mathrm{N}$, Williamson $\mathrm{E}$, Linnane $\mathrm{B}$, et al. Infection, inflammation, and lung function decline in infants with cystic fibrosis. Am J Respir Crit Care Med. 2011;184:75-81.

10 de Jong PA, Nakano Y, Lequin $\mathrm{MH}$, et al. Estimation of lung growth using computed tomography. Eur Respir J 2003;22:235-8.

11 de Jong PA, Long FR, Wong JC, et al. Computed tomographic estimation of lung dimensions throughout the growth period. Eur Respir J 2006;27:261-7.

12 Linnane B, Robinson P, Ranganathan $\mathrm{S}$, et al. Role of high-resolution computed tomography in the detection of early cystic fibrosis lung disease. Paediatr Respir Rev 2008;9:168-74; quiz 74-5.

13 Sly PD, Brennan S, Gangell C, et al. Lung disease at diagnosis in infants with cystic fibrosis detected by newborn screening. Am J Respir Crit Care Med 2009; 180:146-52.

14 Stick SM, Brennan S, Murray C, et al. Bronchiectasis in infants and preschool children diagnosed with 


\section{Editorial}

cystic fibrosis after newborn screening. J Pediatr 2009;155:623-8 e1.

15 Thia LP, Calder A, Stocks J, et al. London cystic fibrosis collaboration. Is chest CT useful in newborn screened infants with cystic fibrosis at 1 year of age? Thorax $2014 ; 69: 320-7$.

16 Wainwright CE, Vidmar S, Armstrong DS, et al. Effect of bronchoalveolar lavage-directed therapy on Pseudomonas aeruginosa infection and structural lung injury in children with cystic fibrosis: a randomized trial. JAMA 2011;306:

163-71.

17 Durmowicz AG, Witzmann KA, Rosebraugh C, et al. Change in sweat chloride as a clinical end point in cystic fibrosis clinical trials: the ivacaftor experience. Chest 2013;143:14-8.

18 Rowe SM, Accurso F, Clancy JP. Detection of cystic fibrosis transmembrane conductance regulator activity in early-phase clinical trials. Proc Am Thorac Soc 2007;4:387-98.
19 Clancy JP, Szczesniak RD, Ashlock MA, et al. Multicenter intestinal current measurements in rectal biopsies from CF and Non-CF subjects to monitor CFTR function. PloS ONE 2013;8:e73905.

20 Cohen TS, Prince A. Cystic fibrosis: a mucosal immunodeficiency syndrome. Nat Med 2012;18: 509-19.

21 Hartl D, Gaggar A, Bruscia E, et al. Innate immunity in cystic fibrosis lung disease. J Cyst Fibros 2012;11:363-82. 